\title{
EL MÉTODO CLÍNICO CENTRADO EN LA PERSONA Y SU APLICACIÓN EN LA ATENCIÓN PRIMARIA DE SALUD
}

\author{
María Sofía Cuba Fuentes ${ }^{1,2, a, b}$, Zoila Olga de los Milagros Romero Albino ${ }^{2,3, a, c}$
}

\begin{abstract}
RESUMEN
El método clínico centrado en la persona (MCCP) aplicado en la atención primaria de la salud (APS) es una herramienta útil para el mejor abordaje de la persona, en el contexto de un sistema de salud que responda de manera adecuada a las necesidades de la población. La APS se constituye en la base de un buen sistema de salud a través del cumplimiento de sus cuatro atributos: primer contacto, longitudinalidad, integralidad y coordinación; y se convierte en parte primordial del crecimiento de cualquier nación que desee brindar una atención de calidad. Concluimos, que el MCCP se relaciona con una APS fuerte, pues se basa en un enfoque de necesidades cubiertas para la población.
\end{abstract}

Palabras clave: Atención Primaria de Salud; Atención dirigida al paciente (Fuente: DeCS BIREME)

\section{THE CLINICAL PERSON-CENTERED METHOD AND ITS APPLICATION IN PRIMARY HEALTHCARE}

\begin{abstract}
The person-centered clinical method (PCCM) used in primary healthcare (PHC) is a useful tool to improve a person's approach to a health system that adequately responds to the needs of the population. PHC is built on the basis of a good health system, through compliance with its four attributes-first contact access, person focused care over time, comprehensiveness, and coordination of care-and it becomes an essential part of the growth of any nation that wants to provide quality healthcare. In conclusion, the PCCM is related to a strong PHC, as it is based on an approach focused on meeting people's needs.
\end{abstract}

Keywords: Primary health care; Patient-centered care (Source: MESH NLM)

\section{INTRODUCCIÓN}

La atención centrada en el paciente ha demostrado generar mejores resultados en la salud de los pacientes ${ }^{(1)}$ y mayor satisfacción en los prestadores de salud (2); sin embargo, su práctica requiere que los profesionales de salud cambiemos nuestro paradigma de salud-enfermedad al de las personas. Además, es necesario que el sistema de salud cumpla con ciertas características que permitan asegurar que los clínicos satisfagan las necesidades del paciente de una manera integral.

En el presente trabajo pretendemos describir el método clínico centrado en la persona (MCCP) en la práctica de la atención primaria de la salud (APS) como herramienta para un mejor abordaje de la persona, en el contexto de un sistema de salud acorde a las necesidades de la población.

\section{ATENCIÓN PRIMARIA EN EL CENTRO DEL SISTEMA}

LaAPS es percibida en el mundo como la base para un buen sistema sanitario que da resultados de salud adecuados ${ }^{(3)}$.
Aunque fue conceptualizada en la Conferencia Internacional sobre Atención Primaria de Salud de Alma-Ata en $1978^{(4,5)}$ e implementada tempranamente por muchos países; fue la doctora Barbara Starfield quien ayudó a perfeccionar su conceptualización y medir su desempeño de acuerdo a cuatro atributos que consideró necesarios para para lograr resultados sanitarios adecuados; además, identificó a la APS como el primer contacto del paciente con el sistema sanitario ${ }^{(6)}$.

Los cuatro atributos de la APS ${ }^{(7)}$ son: el primer contacto y acceso a equipos de atención primaria entrenados, con capacidad resolutiva y tecnología pertinente; longitudinalidad y cupo, personas que desarrollen relación con las familias y comunidades a su cargo, y que tienen responsabilidad por su salud; integralidad, entendida como cobertura de la necesidades de las personas; y coordinación entre los niveles de atención.

Una medicina centrada en las personas requiere de un sistema que facilite el encuentro entre el profesional sanitario de atención primaria y los pacientes. El

\footnotetext{
Dirección General de Promoción de la Salud, Ministerio de Salud. Lima, Perú

Universidad Peruana Cayetano Heredia. Lima, Perú

Seguro Social de Salud del Perú. Lima, Perú

Especialista en Medicina Familiar; ${ }^{\mathrm{b}}$ maestría en Ciencias Clínicas; ${ }^{\mathrm{c}}$ maestría en Geriatría y Gerontología

Recibido: 21/09/2016 Aprobado: 23/11/2016
}

Citar como: Cuba-Fuentes MS, Romero ZO. El método clínico centrado en la persona y su aplicación en la atención primaria de salud. Rev Peru Med Exp Salud Publica. 2016;33(4):780-4. doi: 10.17843/rpmesp.2016.334.2565 
cumplimiento de los atributos de la APS es la meta para cualquier sistema sanitario y es el espacio en donde se puede conseguir que el foco sean las personas.

\section{LA MELODÍA DEL PACIENTE}

Luego de ver una partitura musical por primera vez, es posible que el aprendiz haya observado solo símbolos carentes de un significado; sin embargo, con el pasar del tiempo, el entrenamiento ayuda a reconfigurar la simbología, otorgándole un significado que, en suma, dará como fruto una melodía. Si trasladamos esta analogía a nuestra práctica diaria -comparando a la partitura con el paciente-, ese conjunto de símbolos extraños que constituyen la partitura se asemejan a los signos o síntomas de nuestros pacientes; "símbolos" que a medida en que trascurre el entrenamiento o el contacto con las personas, van tomando sentido y se transforman en una melodía que nos permite comprender mejor el estado de salud y ser capaces de resolver sus problemas ${ }^{(8)}$.

\section{MÉTODO CLÍNICO CENTRADO EN LA PERSONA}

Una mirada hacia la atención sanitaria que actualmente se brinda en los diversos establecimientos de salud de nuestro país, nos motiva a presumir que la mayor parte de profesionales de salud tienen como punto de partida a la enfermedad al momento de tratar de resolver el complejo mundo que constituye cada persona; observación que es similar a lo descrito por lan McWhinney en $1994^{(9,10)}$.

Entender a las personas en su contexto y explorar el significado que tiene la enfermedad en la vida de la persona son parte de los cambios que se plantean en la construcción del nuevo paradigma ${ }^{(11)}$; entendiendo que la enfermedad, persona y entorno no son independientes, sino que confluyen ${ }^{(12)}$.

Para comprender estas acepciones discutiremos el método clínico centrado en la persona desarrollado por Ian McWhinney, Moira Stewart y Judith Brown en Canadá, quienes se basaron en el conocimiento de que el paradigma existente no respondía las necesidades de las personas ${ }^{(13)}$.

Este método clínico busca que los profesionales de la salud aborden cuatro componentes cuando se aproximan a las personas: abordar la dolencia, la salud y la enfermedad; aproximarse a la persona como un todo; llegar a un acuerdo común en base a la definición de problemas, objetivos y roles; y desarrollar la relación médico-paciente (Figura 1).
ABORDAR LA DOLENCIA, LA SALUD Y LA ENFERMEDAD

El MCCP nos permite abordar la complejidad del paciente ${ }^{(14)}$. En la práctica diaria, dadas las condiciones del sistema de salud y de la formación recibida tanto en pregrado como en posgrado, los profesionales de la salud están entrenados para diagnosticar y tratar la enfermedad. Por tal motivo, los pacientes, sintomáticos o no, son categorizados de acuerdo a algún problema de salud que podría o no estar interfiriendo con su desempeño; explorando poco o nada su dolencia, entendida esta última como aquellos sentimientos, ideas, expectativas que alteran su función, y están relacionadas con la enfermedad o síntoma. Es necesario precisar que las personas no siempre se encontrarán en alguno de estos grandes grupos (enfermedad o dolencia), sino en su gran mayoría, en una combinación de los mismos. La salud puede ser concebida como la capacidad para cumplir sus propósitos y metas; y estará muy relacionada a los valores y aspiraciones de cada persona ${ }^{(15)}$.

En ese sentido, al explorar la enfermedad, debemos evaluar los síntomas, los resultados del examen físico y los exámenes de ayuda diagnóstica; al explorar la dolencia debemos indagar sobre cuatro puntos cruciales: los sentimientos del paciente acerca del problema, las ideas que tienen acerca del problema; el efecto de estas dolencias en su funcionamiento diario, sus expectativas con respecto al médico que los atiende y finalmente, al explorar la salud debemos tener en cuenta la percepción única y la experiencia de salud que tiene cada paciente ${ }^{(16)}$.

\section{APROXIMARNOS A LA PERSONA COMO UN TODO}

El carácter y la personalidad, contextualizados con la experiencia vivida y formación, son los que influyen en el estado evolutivo en el cual se encuentra la persona y definen mucho su comportamiento ${ }^{(8,17)}$. El profesional de la salud debe tener en cuenta la parte espiritual del paciente, comprender sus distintas creencias y religiones, y por tanto, escucharlos con sinceridad, respeto, aceptación y sin prejuicios.

Dentro del contexto de la persona podemos hablar de dos tipos: próximo y remoto. Al hablar del contexto próximo nos referirnos, en primera instancia, a la familia, examinando el papel y la influencia que esta ejerce en el paciente; además, la respuesta del paciente a la experiencia de la enfermedad. Ayuda a ampliar la perspectiva del profesional de la salud en cómo la situación podría evolucionar: una enfermedad puede alterar el concepto de apoyo, confianza y hasta de amor familiar; es así que cuando una dolencia o una enfermedad afectan a algún miembro, se desequilibra la armonía de la familia y puede, incluso, conducir posteriormente a una mala dinámica familiar (17). Además de la familia, se deben evaluar otros contextos 
1. Explorando dolencia y enfermedad

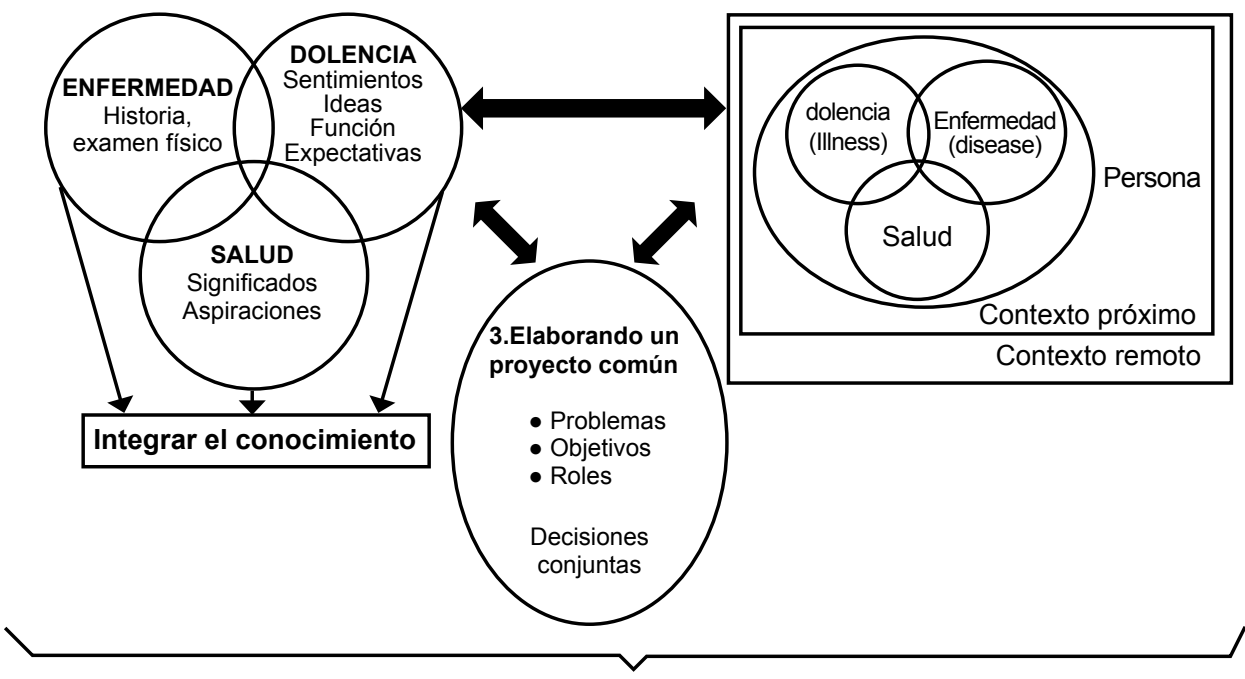

4. Intensificando la relación médico paciente

Fuente: Adaptado de Stewart M, et.al. Patient-centered clinical medicine, Transforming the Clinical Method. 3ra edición. New Zealand: CRC Press; 2014.

Figura 1. Método clínico centrado en las personas y sus cuatro componentes

proximales influyentes en la homeostasis del individuo en cuanto a su salud y enfermedad ${ }^{(18)}$.

Otro de los componentes que es importante evaluar, es el contexto remoto que influye sobre la persona. El contexto remoto se constituye, básicamente, por los determinantes de la salud del individuo tales como la comunidad, la cultura, la economía, el sistema de salud, los factores socioculturales, la geografía, los medios de comunicación y el ecosistema de salud ${ }^{(19)}$. Todos estos aspectos en poca, mediana o gran magnitud influyen en la persona.

En ese sentido, es pertinente concluir en este acápite, que para una exploración adecuada de la persona se debe tener en cuenta la exploración del desarrollo individual y espiritual de la persona a través de las diferentes etapas del ciclo evolutivo, la familia como contexto próximo y la comunidad en toda su acepción, como contexto remoto.

\section{LLEGAR A UN ACUERDO COMÚN, DEFINICIÓN DE PROBLEMAS OBJETIVOS Y ROLES}

Constituye el punto más crucial de la interacción médicopaciente. En este componente se establecen pactos entre ambos participantes de la entrevista, por lo que se debe definir el problema, establecer metas y prioridades del tratamiento, así como definir y asignar los roles tanto del médico como del paciente.

Según Lawrence Weed: Problema es todo aquello que requiere diagnóstico, manejo posterior, o que interfiere con la calidad de vida, de acuerdo con la percepción del paciente ${ }^{(20)}$. Una vez identificado el problema, se procede al desarrollo de las metas y prioridades del tratamiento, para lo cual es imprescindible que el médico sea realista y no exija algo que esté fuera del alcance del paciente; pues medidas inalcanzables podrían llevar a un mayor incumplimiento al tratamiento y frustración tanto del paciente como del médico ${ }^{(21)}$

Adicionalmente, es necesario asignar roles tanto al médico como al paciente considerando las capacidades de los participantes en la entrevista clínica. Las claves para alcanzar exitosamente la meta son: evitar la imposición de los deberes desde un único polo de la entrevista y motivar el cambio basado en las ideas e intereses del paciente, forjando una actitud de autocuidado en función de la información facilitada por el médico y contrastada en mayor o menor medida con los conceptos interiorizados a lo largo de la vida del paciente ${ }^{(22-24)}$.

Finalmente, se encuentra el componente de la "entrevista clínica" que integra los otros tres componentes, en donde la valoración y apreciación de la opinión y emoción de los pacientes lograrán la confianza y convicción que se requiere para abordar sus problemas de forma idónea. En este punto, es crucial valorar la importancia de la toma de decisiones a través de sus cuatro modelos: el paternalista, la decisión informada, el médico como agente perfecto, y la toma de decisiones compartidas (shared decision making).

En la toma de decisiones compartidas, pacientes y médicos se enfrentan a situaciones de incertidumbre, donde no hay un tratamiento "evidentemente superior", debiéndose tomar una decisión sobre múltiples opciones, inciertas en beneficios, efectos adversos y costos de 
diferente valor individual, salvaguardando y promoviendo los derechos fundamentales de los pacientes ${ }^{(25)}$.

\section{DESARROLLAR LA RELACIÓN MÉDICO PACIENTE}

Este cuarto componente demanda una responsabilidad compartida de poder y control entre médico y paciente ${ }^{(26)}$. Una buena relación médico-paciente requiere una alianza de trabajo o terapéutica, así como de habilidades tan variadas que van desde el uso de la tecnología, la perspicacia psicológica y las comunicativas, como es la empatía. De acuerdo con Cassell: El primer escalón en el tratamiento es el médico ${ }^{(26)}$.

Cada relación que se establece es única, y puede variar según el tipo de paciente. Los médicos tienen la habilidad de desarrollar diferentes papeles de acuerdo al tipo de paciente que traten; a veces pueden ser un instructor, otras veces el guía, otras un familiar y otras, el médico ${ }^{(27)}$. Dentro de las condiciones que ayudan a afianzar esta relación médico paciente-encontramos ${ }^{(17,25,26) \text { : }}$

Compasión, cuidado, empatía y confianza. Es imprescindible comprender que nuestra dificultad consiste en que fallamos en entender lo que el paciente quiere, que algunas veces puede ser algo muy simple: reconocer su sufrimiento, o quizá nuestra compañía en el momento de la necesidad.

Continuidad y constancia. Este aspecto está supeditado en algunas ocasiones por las decisiones políticas, ocasionado un resquebrajamiento en la continuidad del cuidado de la salud; el médico, en este caso, se enfrenta a diversas situaciones como por ejemplo un paciente con una conducta difícil, malestar por el sistema de salud, etc.

Curación y esperanza. Desde la perspectiva del médico, este debe entender que todo sufrimiento es único e individual, involucra el autoconflicto y está marcado por la pérdida del propósito central que es atender a la persona que fue antes de la enfermedad y que no toda esperanza está perdida; desde la perspectiva del paciente es ser alentado por el médico a combatir su enfermedad, por lo que se seguirán ciertos pasos en el proceso de curación y en donde se demandarán expectativas distintas, desde escuchar, trasmitir confianza, incentivar voluntad de cambio e, incluso, adquirir nuevas habilidades para la vida.

Autoconciencia y sabiduría práctica. Se sugiere que los médicos tengan una postura más receptiva (escucha activa) y pensamiento más analítico a favor de un proceso más contemplativo, sobre todo cuando estamos ante un paciente que "no es de nuestro agrado"; se trata de replantear la mentalidad previamente concebida y prestar atención a nuestras palabras, gestos y presentación para con el paciente, a fin de entenderlo y poder ayudarlo.
Transferencia y contratransferencia. La transferencia es un fenómeno que está presente en la vida cotidiana y sucede de forma inconsciente; sucede cuando el paciente trasmite sus vivencias pasadas como amor, odio, ambivalencia y dependencia, y mientras más significativa sea la relación médico-paciente será más probable que ocurra este fenómeno. La transferencia puede ser percibida como elemento negativo; sin embargo, puede ayudar a conseguir la conexión que se requiere entre el paciente y el médico ${ }^{(27,28)}$.

La contratransferencia ocurre cuando el médico responde frente a la transferencia del paciente de un modo similar a relaciones pasadas significativas. El médico debe saber qué es lo que le están transfiriendo y cómo tiene que responder; ser consciente y devolver la responsabilidad al paciente. A veces la contratransferencia se determina en el momento en el que existe conexión empática en el encuentro médico-paciente, en otras ocasiones es más elusivo al desconocimiento médico ${ }^{(29)}$.

\section{DISCUSIÓN}

Los cuatro atributos que estableciera la doctora Barbara Starfield para la APS se han constituido en la base de un buen sistema sanitario, adecuado a las necesidades de la población. En nuestro país, a raíz de la reforma en salud, se han establecido una serie de pautas que podrían confluir en la constitución de una atención primaria fuerte; sin embargo, para que esto pueda concretarse, los profesionales de la salud, además de ser adecuadamente remunerados y tener buenas condiciones para la prestación de la salud, deben poseer competencias que le permitan acercarse a las personas para la resolución de sus problemas más frecuentes.

En ese sentido, el MCCP se constituye en una herramienta fundamental que debería formar parte de la experticia de todos los profesionales de la salud que brindan atención en el primer nivel de atención de salud; este método se basa en el abordaje de cuatro componentes importantes, que desde la concepción de la dolencia, enfermedad y salud; la aproximación de la persona en su contexto proximal y distal; la toma de decisiones compartidas; hasta el desarrollo de una adecuada relación médico-paciente, se constituyen en la base para que los atributos de la atención de la APS puedan sedimentarse.

Es importante internalizar la idea de que mientras más tempranamente los profesionales de la salud comprendan la significancia del MCCP, el abordaje hacia la persona será mucho más fluido; es por ello que, apostar por su desarrollo desde el pregrado forma uno de los objetivos que debemos concretar en un espacio donde confluyen no solo elementos desde salud, sino también desde educación.

Ciertamente, existe una brecha que es necesario recorrer y en el que se encuentran implicados varios 
actores para la consolidación de una APS fuerte, por lo que se requiere crear las condiciones propicias con el fin de consolidar la propuesta.

\section{CONCLUSIONES}

La atención primaria de salud es, entonces, la base de un buen sistema de salud, por lo que apostar por la operativización de sus cuatro atributos es fundamental en el abordaje de las personas. EI MCCP debe formar parte de las competencias de los profesionales de la salud y ser internalizado desde fases tempranas de su desarrollo, a fin de concretar los atributos que definiera la doctora Barbara Starfield. Se debe consensuar una propuesta multisectorial que permita garantizar una adecuada salud para todos los peruanos desde diversos ámbitos de acción.

Fuentes de financiamiento: autofinanciado.

Conflictos de interés: los autores declaran no tener conflictos de interés.

Contribución de autoría: MCF y ZRA participaron en la concepción, redacción, revisión y aprobación de la versión final del manuscrito.

\section{REFERENCIAS BIBLIOGRÁFICAS}

1. Stevenson FA, Cox K, Britten N, Dundar Y. A systematic review of the research on communication between patients and health care professionals about medicines: the consequences of concordance. Health Expect. 2004;7(3):235-45. doi: 10.1111/j.1369-7625.2004.00281.x

2. Dwamena F, Holmes-Rovner M, Gaulden CM, Jorgenson S, Sadigh G, Sikorskii $A$, et al. Interventions for providers to promote a patient-centred approach in clinical consultations. Cochrane Database Syst Rev. 2012;12:CD003267. doi: $\quad 10.1002 / 14651858 . C D 003267$. pub2

3. Starfield B. New paradigms for quality in primary care. $\mathrm{Br} \mathrm{J}$ Gen Pract. 2001;51(465): 303-9.

4. Organización Mundial de la Salud. Declaración de Alma Ata. Kazajstán: Organización Mundial de la Salud; 1978.

5. Informe sobre la salud en el mundo 2008: la atención primaria de salud, más necesaria que nunca. Ginebra: Organización Mundial de la Salud; 2008.

6. Starfield B, Gérvas J, Mangin D. Clinical care and health disparities. Annu Rev Public Health. 2012; 33: 89-106. doi: 10.1146/annurevpublhealth-031811-124528

7. Starfield B. Atención primaria: equilibrio entre necesidades de salud, servicios y tecnología. 2a ed. Madrid: Masson; 2004.

8. Stewart M, Belle J, Wayne W, McWhinney I, McWilliam C, Freeman T. Patient-centered medicine: transforming the clinical method. 3a ed. New York: Radcliffe Publishing Ltd; 2014.

9. McWhinney I. Medicina de familia. Barcelona: Mosby/Doyma; 1994.

10. Mezzich JE. Repensando el centro de la medicina: de la enfermedad a la persona. Acta Méd Peruana 2010;27(2):148-50. 2010.
11. McWhinney I, Freeman T. Textbook of Family Medicine. 3a ed. New York: Oxford University Press, Inc.; 2009.

12. Cassell EJ. The Healers Art: A New Perspective on the Doctor-Patient Relationship. 1a ed. New York: Lippincott; 1976.

13. Hinds PS, Chaves D, Cypess S. Context as source of meaning and understanding. Qual Health Res. 1992. 2(1):42-60. doi: $10.1177 / 104973239200200105$

14. Brown J, Stewart M, Tessier S. Assessing communication between patients and doctors: a manual for scoring patientcentred communication. London: Thames Valley Family Practice Research Unit; 1995.

15. Commission on Social Determinants of Health. Closing the gap in a generation: health equity through action on the social determinants of health. Final report. Geneva: World Health Organization; 2008.

16. Saéz M. Modelo clínico centrado en el paciente. Archivos de Medicina Familiar y General. 2008;5(1):2-11.

17. Tizón-García JL. A propósito del modelo biopsicosocial, 28 años después: epistemología, política, emociones y contratransferencia. Aten Primaria. 2007;39(2):93-7. doi: $10.1157 / 13098677$

18. Membrillo A; Fernández M, Quiroz J, Rodríguez J. Familia. Introducción al estudio de sus elementos. México: ETM; 2008.

19. Suarez M. El genograma: herramienta para el estudio y abordaje de la familia. Rev Méd La Paz. 2010;16(1): 53-57

20. Savage P. Problem oriented medical records. BMJ. 2001;322(7281):275.

21. Martínez, T. La atención gerontológica centrada en la persona. Guía para la intervención profesional en centros y servicios de atención a personas mayores en situación de fragilidad o dependencia. Vitoria-Gasteiz: Servicio Central de Publicaciones del Gobierno Vasco; 2011.

22. Cooper AF. Whose illness is it anyway? Why patient perceptions matter. Int J Clin Pract. 1998;52(8): 551-6.

23. Quill TE, Brody $H$. Physician recommendations and patient autonomy: finding a balance between physician power and patient choice. Ann Intern Med. 1996;125(9):763-9.

24. Beaulieu MD. President's message: toward a patient-centred health care system. Can Fam Physician. 2013;59(1): 109-10.

25. Powers BW, Jain SH. Patient-centered physician selection: a necessary first step for accountable care. Am J Manag Care. 2014;20(11 Spec No.): E16

26. Cassell EJ. The Nature of Healing: The Modern Practice of Medicine. 1a ed. New York: Oxford University Press, Inc.; 2012.

27. Scott JG, Cohen D, Dicicco-Bloom B, Miller WL, Stange KC, Crabtree BF. Understanding healing relationships in primary care. Ann Fam Med. 2008;6(4):315-22. doi: 10.1370/afm.860

28. Tulsky JA. Interventions to enhance communication among patients, providers, and families. J Palliat Med. 2005;8(Suppl 1):S95-102. doi: 10.1089/ jpm.2005.8.s-95

29. Sociedad Española de Medicina de Familia y Comunitaria. Tratado de medicina de familia y comunitaria. 2a ed. Madrid: Editorial Médica Panamericana S.A.; 2012.

Correspondencia: Maria Sofia Cuba Fuentes Dirección: Calle José Gonzales 775, departamento 604. Miraflores, Lima, Perú. Teléfono: (+511) 958914161

Correo electrónico: sofiacuba@hotmail.com 